\title{
171. On the Relation between Tannin Vacuoles and Protoplasm in the Motor Cell of Mimosa pudica L. ${ }^{*}$
}

\author{
By Hideo Toriyama**) \\ (Comm. by Tetsu Sakamura, m.J.A. Oct. 12, 1967)
}

In the previous paper, the results of study on the tannin vacuoles and protoplasm in the motor cell of Mimosa pudica in both diurnal and nocturnal conditions were briefly reported (Toriyama, 1967a). It has been showed that before receiving a stimulus, tannin vacuoles were not stained with alcoholic toluidine blue solution, while, after stimulation, they were stained blue very strongly with the same dye. This finding about the change of the affinity of tannin vacuole for the dye, provides an important clue to the solution of the problem on the mechanism in the motor cell of Mimosa. In another paper, appeared in the same year, the author (Toriyama, 1967b) has reported that, by the application of the Kaiser-toluidine blue technique, protoplasm was found mixed with tannin after stimulation. In the present investigation, the further observations on the interaction of tannin vacuole and protoplasm are done, which may serve to the elucidation of the mechanism of the motor cell. In the previous work, it was made possible to distinguish the stimulated material from the non-stimulated, based on the affinity of tannin vacuole for Ehrlich-Haematoxylin and toluidine blue. In order to confirm the special affinity of tannin substance for the basic dyes, which may possibly be avairable to distinguish it from protoplasm, several kinds of the dye, other than toluidine blue, were proved now.

The materials used here, were similar to those in the previous works (Toriyama, 1967a, 1967b). The motor tissue of the main pulvinus before and after stimulation in the diurnal period, came to the special consideration. In order to get the motor cell materials before the bending movement, the plants were exposed to ether vapour. The fixing methods were applied in this case too. In addition, a new technique was adopted to facilitate the observation on the structure of tannin portion in relation to protoplasm in the motor cells.

Experimental results. The materials of the motor cells were

*) Contribution No. 48 from the Biological Section, Tokyo, Woman's Christian College. This research has been supported in part by a Grant-in-Aid for Scientific Research from the Ministry of Education.

**) Biological Section, Tokyo Woman's Christian College, Tokyo. 
fixed with Müller's fluid*) and/or Zenker's fluid.**) The washing, dehydration and cutting procedure of the materials were performed in the same manner as in the previous paper (Toriyama, 1967a). Both materials, before and after stimulation were stained with 0.05 per cent aqueous solution of azur II, methylen blue, toluidine blue, brilliant cresyl blue, Nile blue, gentian violet, methyl blue, aniline blue, and Mallory's aniline blue-orang $G^{* * *}$ and also Ehrlich-Haematoxylin respectively. The affinity of the tannin vacuole for these dyes is shown in Table I.

Table I. Affinity of tannin vacuole for several dyes

\begin{tabular}{l|c|c|c|c}
\hline \multicolumn{1}{c|}{ Fixatives } & \multicolumn{2}{|c|}{ Müller's fluid } & \multicolumn{2}{c}{ Zenker's fluid } \\
\cline { 2 - 5 } \multicolumn{1}{c|}{ Stimulation } & before & after & before & after \\
\hline Azur II (B) & \pm & ++ & \pm & + \\
Methylen blue (B) & - & ++ & - & +++ \\
Toluidine blue (B) & \pm & +++ & - & +++ \\
Brilliant cresyl blue (B) & - & ++ & - & + \\
Nile blue (B) & - & ++ & - & ++ \\
Gentian violet (B) & - & ++ & - & - \\
Methyl blue (A) & - & \pm & - & - \\
Aniline blue (B) & - & + & - & ++ \\
Mallory's staining & - & - & - & - \\
Ehrlich-Haematoxylin & - & +++ & - & - \\
\hline
\end{tabular}

+ indicates staining reaction.

(B): basic dye; (A): acid dye.

In the material which was fixed with Müller's fluid or Zenker's fluid, tannin vacuoles were found not stained by the several basic dyes before the stimulation. On the contrary, after the stimulation, tannin vacuoles were stained strongly. For instance, the Zenkertoluidine blue technique made it possible clearly to distinguish the stimulated material from the non-stimulated (Table I, Figs. 1a and 1b). The same results can be attained with other basic dyes. Two exceptional cases were met with methyl blue (acid dye) and Mallory's staining, which could not show the difference between the stimulated and the non-stimulated.

For the first time it was found by the present author, that some organic acid, especially glacial acetic' acid $\left(\mathrm{GAA}^{* * * *)}\right)$, remarkably affects the affinity of tannin vacuole for the dyes. A series of experi-

*) Müller's fluid is composed of $2.5 \mathrm{~g}$ of potassium bichromate, $1 \mathrm{~g}$ of sodium sulphate, and distilled water $(100 \mathrm{ml})$.

**) Zenker's fluid is composed of $100 \mathrm{ml}$. of a $2.5 \%$ potassium bichromate, $5 \mathrm{~g}$ of mercuric chloride, $5 \mathrm{ml}$. of glacial acetic acid.

$* * *)$ Cited from Romeis, B. (1932).

****) This abridged form will be used in the following lines. 
on this noticeable effect of GAA have been carried out using materials, similar to those in the foregoing experiments. Both materials, before and after the stimulation, were fixed with Zenker's fluid for 10 hours. The procedure of the preparation was as usual, except that after the washing with distilled water the sections on the slide glass were soaked in 5 per cent GAA $30^{\circ} \mathrm{C}$, for 5 minutes to 24 hours. After this procedure the sections were washed in distilled water thoroughly, and stained with 0.05 per cent solution of several basic dyes, namely, Nile blue, brilliant cresyl blue, methylen blue, toluidine blue, and aniline blue respectively. The affinity of tannin vacuoles for the several dyes is shown in Table II.

Table II. Effect of glacial acetic acid on affinity of tannin vacuole for basic dyes in the cells were fixed with Zenker's fluid

\begin{tabular}{|c|c|c|c|c|c|c|c|c|c|c|}
\hline \multirow[b]{2}{*}{$\begin{array}{l}\text { Treat- } \\
\text { ment time } \\
\text { in min. }\end{array}$} & \multicolumn{5}{|c|}{ Before stimulation } & \multicolumn{5}{|c|}{ After stimulation } \\
\hline & N.B. & M.B. & B.C.B. & T.B. & A.B. & N.B. & M.B. & B.C.B. & T.B. & A.B. \\
\hline 0 & - & - & - & - & - & ++ & +++ & + & +++ & ++ \\
\hline 5 & - & ++ & + & + & + & - & \pm & \pm & \pm & + \\
\hline 10 & - & + & + & + & ++ & - & + & \pm & \pm & + \\
\hline 20 & - & + & + & + & - & \pm & + & \pm & + & + \\
\hline 30 & ++ & ++ & ++ & ++ & - & + & + & + & + & \pm \\
\hline 60 & +++ & +++ & +++ & +++ & ++ & + & + & + & + & \pm \\
\hline 90 & +++ & +++ & +++ & +++ & +++ & \pm & \pm & \pm & \pm & - \\
\hline 120 & +++ & +++ & +++ & +++ & +++ & - & - & - & - & \pm \\
\hline 240 & +++ & ++ & +++ & +++ & +++ & + & + & + & + & + \\
\hline 300 & +++ & +++ & +++ & +++ & +++ & $+\quad *$ & $+*$ & $+*$ & $+*$ & $+*$ \\
\hline 1080 & +++ & +++ & +++ & +++ & +++ & $++*$ & $++*$ & $++*$ & $++*$ & $++*$ \\
\hline 1440 & +++ & +++ & +++ & +++ & +++ & $+++*$ & $+++*$ & $+++*$ & $+++*$ & $+++*$ \\
\hline
\end{tabular}

N.B., Nile blue; M.B., Methylen blue; B.C.B, Brilliant cresyle blue; T.B, Toluidine blue; A.B., Aniline blue. + indicates staining reaction; * indicates the appearence of bead-like threads.

In these materials, there were recognized some differences between the stimulated cell and the non-stimulated, concerning to the affinity of the tannin vacuole for the basic dyes. It is worthy of mentioning that this nature of the tannin vacuole was affected by the GAA-treatment. Now it is noticeable that the affinity of tannin vacuole for the basic dyes, which was not found in the nonstimulated material, appeared but by the GAA-treatment, and became stronger and stronger with progress of this treatment $(30 \sim 1440$ minutes). On the contrary to the non-stimulated material mentioned above, the affinity of tannin vacuole, which had appeared directly after the stimulation, gradually decreased during the GAA-treat- 

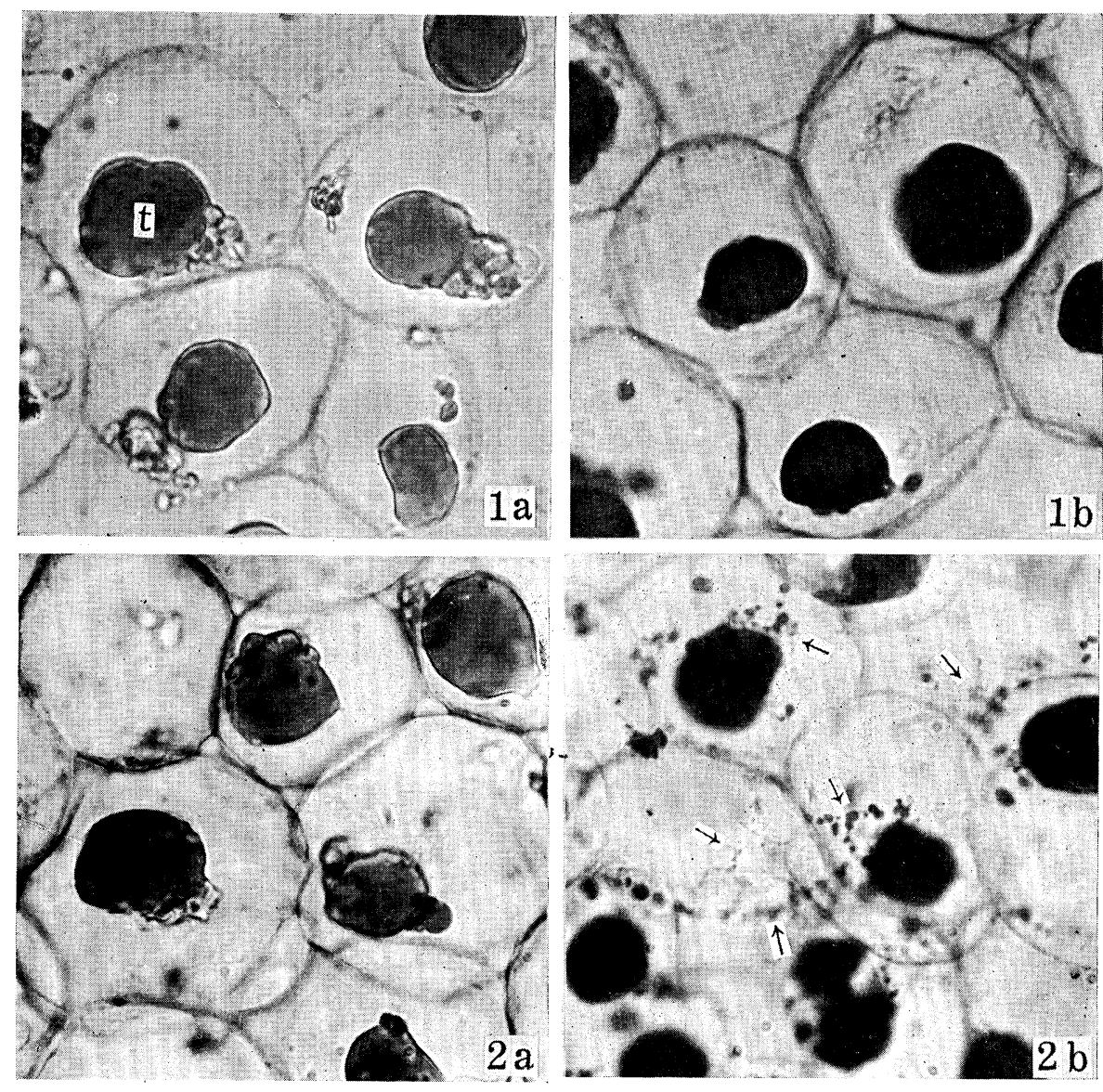

Figs. 1a-1b. The motor tissue-cells of the main pulvinus of Mimosa pudica. $1 \mathrm{a}$, before stimulation. $t$, tannin vacuole; $1 \mathrm{~b}$, after stimulation. $\times 900$. The above two cell groups originate from the material fixed with Zenker's fluid and stained with toluidine blue. 2a, before stimulation; $2 b$, after stimulation. Arrows indicate bead-like thread. $\times 900$. The above two cell groups originate from the material fixed with Zenker's fluid and treated with glacial acetic acid for 1080 minutes and stained with toluidine blue.

ment, and was perfectly lost after the treatment of 120 minutes. By the longer treatment with the same acid, however, this affinity was recovered again, until the strongst staining was attained in the material of the 1440 minutes GAA-treatment.

From the available data of Table II, it is difficult to explain the chemical relation between tannin vacuole and the basic dyes. However, it may be said that this data, obtained by the special new method, indicate the more notable differences of the nature of tannin vacuole, between the stimulated and non-stimulated motor cell.

By the GAA-treatment, also some following cyto-morphological 

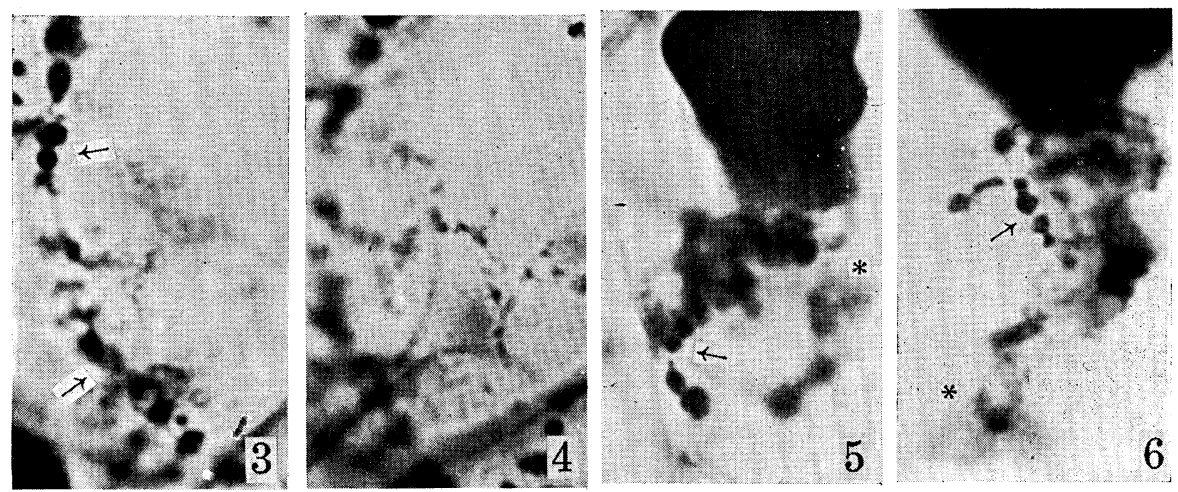

Figs. 3-6. The bead-like threads of tannin in motor cell of Mimosa pudica after stimulation. Arrows indicate bead-like thread; asterisks indicate diffused type of tannin mixing. $\times 1500$. The above four cells originate from the material fixed with Zenker's fluid and treated with glacial acetic acid for 1080 minutes and stained with toluidine blue.

data were observed: before receiving a stimulus, tannin vacuoles in the materials treated with GAA for 1080 minutes, were showed in Fig. 2a. The tannin vacuole appeared very clearly and no other stained figure of tannin were visible. After the stimulation, the contours of the tannin vacuoles became obscure, and the beadlike thread appeared, which were stained blue by the dye with the same treatment (Fig. 2b). In these preparations, though hardly caught in the microphotographs, the fine bead-like thread is easily observable (Fig. 4). The threads which appear in the stimulated materials may be roughly classified into three categories; namely, bead-like form (shown by arrows in Figs. 3, 5, and 6), fine beadlike thread (Fig. 4), and diffused type (shown by asterisks in Figs. 5 and 6). It is worth while to mention that the threads and tannin vacuoles are both stained with toluidine blue, and no distinct difference in the intensity of color is seen between these two elements of tannin. For this reason, the author tends to consider that the substance in the bead-like threads and the tannin vacuole may probably be of the same origin. In this peculiar figure, a small amount of tannin seems to be contained and it may be produced by the excitation of protoplasm as the result of stimulation. These bead-like threads do not appear in the motor cell which is not stimulated, by the same GAA-treatment (Fig. 2a). If Fig. 2b is compared with Fig. 1b, it seems that the bead-like threads may appear as a result of the separation of tannin from protoplasm, by a certain unmasking action of GAA.

It may be probable that a part of protoplasmic compornent, which has masked the bead-like threads of tannin is removed by 
the treatment of GAA. Thus the bead-like threads are put in appearance, but under these conditions (Figs. 2b, 3, 4, 5, and 6). This treatment, which has been carried out for the first time in the present work, can serve as a new method to make visible the cytological change caused by the stimulation (Fig. 1b). The size reduction of tannin vacuoles (Figs. $1 \mathrm{~b}$ and $2 \mathrm{~b}$ ) makes it probable that the tannin substance comes out from its vacuole and infiltrates into the protoplasm in branching form, as indicated schematically (Fig. 7b). The fine branching tannin thread imbeded in the protoplasm may probably change its own figure into the bead-like thread by the effect of fixatives, as shown in the schema (Fig. 7c).

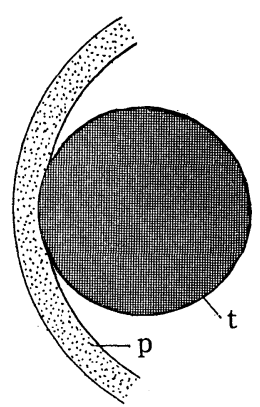

a

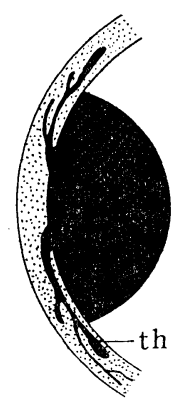

b

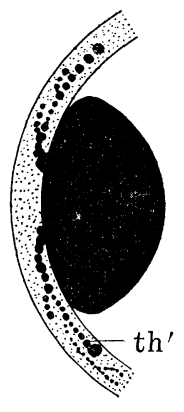

$\mathrm{c}$
Fig. 7. Schematic figure of change in motor cell of the pulvinus of Mimosa pudica. a, before stimulation; $b$, after stimulation; c, after stimulation. Figured according to the fixed materials; $p$, protoplasm; $t$, tannin vacuole. th, thread of tannin; $t^{\prime}$, bead-like thread.

We have briefly stated in the previous paper that tannin vacuoles have physiological significance in the control of the colloidal state of the protoplasts of the motor cells (Toriyama, 1953). It may be postulated that one part of tannin vacuole and a protoplasmic component, probably its protein, are mixed with each other when the motor cell is excited by stimulus. It is probable that the tannin acts to change the colloidal state of protoplasm in the motor cell, and this may cause the increase of the permeability of the protoplasm for potassium.*) The physicochemical nature of the "biological tanning" in the motor cell is expected to be studied in detail in future.

The writer wishes to express his thanks to Miss Yôko Fujita and Miss Masako Iwatsuki for their major assistance in carrying out the experiments.

\section{References}

Romeis, B. (1932): Taschenbuch der mikroskopichen Technik.

Toriyama, H. (1953): Cytologia, 18, 283-292.

- (1955): Cytologia, 20, 367-377.

- (1967a): Proc. Japan Acad., 43, 541-546.

(1967b): Proc. Japan Acad., 43, 384-388.

*) For further references on the potassium migration in Mimosa motor cell, readers are referred to Toriyama (1955). 